STUDI

FRANCESI

\section{Studi Francesi}

Rivista quadrimestrale fondata da Franco Simone

190 (LXIV | I) | 2020

Varia - fasc. I - gennaio-aprile 2020

\title{
Le déguisement et ses miroirs. Histoire et souvenirs dans les "Mémoires" de l'abbé de Choisy
}

\section{Alex Bellemare}

\section{(2) OpenEdition}

1 Journals

\section{Édition électronique}

URL : https://journals.openedition.org/studifrancesi/22119

DOI : 10.4000/studifrancesi.22119

ISSN : 2427-5856

Éditeur

Rosenberg \& Sellier

\section{Édition imprimée}

Date de publication : 1 avril 2020

Pagination : 126-138

ISSN : 0039-2944

\section{Référence électronique}

Alex Bellemare, "Le déguisement et ses miroirs. Histoire et souvenirs dans les "Mémoires" de l'abbé de Choisy », Studi Francesi [En ligne], 190 (LXIV | I) | 2020, mis en ligne le 01 avril 2021, consulté le 02 août 2021. URL : http://journals.openedition.org/studifrancesi/22119; DOI : https://doi.org/10.4000/

studifrancesi.22119

\section{(c) (i) $\odot$}

Studi Francesi è distribuita con Licenza Creative Commons Attribuzione - Non commerciale - Non opere derivate 4.0 Internazionale. 


\section{Le déguisement et ses miroirs. Histoire et souvenirs dans les "Mémoires" de l'abbé de Choisy}

\section{Abstract}

In the Mémoires pour servir à l'bistoire de Louis XIV and the Mémoires de l'abbé de Choisy babille en femme, the abbot of Choisy, who proposes to give an account of his life as well as drawing a portrait of Louis XIV, mobilizes different regimes of memory. Hybridizing fictionnal and real history, while intertwining historical and subjective time, Choisy underlines the boundaries that traditionally separate personal memory and national history. The memorialist's project appears both ambiguous and scandalous, as the story of the Sun King's exploits coexists with autobiographical elements detailing the abbot's transvestite adventures. This article shows that the experience of memory and that of identity are bound under the sign of the travesty, which is a means of dissimulation and unveiling of oneself.

L'abbé de Choisy a porté plusieurs masques. À la fois historien appliqué de l'Église ${ }^{1}$, élu doyen à l'Académie française, coadjuteur de l'ambassade du roi au Siam, libertin intrigant converti au seuil de sa vie, l'abbé de Choisy a légué une œuvre prolixe et disparate, ayant pratiqué les genres les plus variés. Le diptyque constitué des Mémoires pour servir à l'bistoire de Louis XIV et des Mémoires de l'abbé de Choisy babillé en femme $e^{2}$, échafaudé a posteriori par la critique malgré des poétiques parfois rivales $^{3}$, met en œuvre une conception insolite et personnelle de l'histoire. Loin du

(1) F.-T. de Choisy, Histoire de l'Église, Paris, Jean-Baptiste Coignard, Antoine Dezallier \& Christophe David, 1703-1723.

(2) F.-T. de Choisy, Mémoires de l'abbé de Choisy, éd. G. Mongrédien, Paris, Mercure de France, 2000 [1727-1742]. Toutes les références renvoient à cette édition. Les Mémoires pour servir à l'bistoire de Louis XIV paraissent en 1727, tandis que les Mémoires de l'abbé de Choisy babillé en femme sont aussi publiés de manière posthume, mais par morceaux successifs et indépendants, en 1735 d'abord, puis en 1742 , avant que l'édition moderne ne procède à l'assemblage de ces fragments en un texte unitaire.

(3) La paternité de ces deux textes, que l'édition a rassemblés tout en reconnaissant parfois l'absence de cohésion interne, a été sujette à de nombreux débats depuis l'article de J.-Y. Vialleton, La nouvelle diffamatoire dans la France de l'âge classique. Le cas particulier de "La Vie de Monsieur l'abbé de Choisy", article électronique, "Cahiers d'études italiennes" 10, http://cei.revues.org/175. Vialleton, même s'il ne cherche pas à «établir la fausseté de la vie de Choisy telle que la racontent les biographes encore aujourd'hui» (ibidem, par. 8), estime quand même que «ce n'est pas l'œuvre de Choisy qui va assurer sa fortune posthume, mais l'image peu enviable que va lui construire la postérité en lui attribuant d'autres ouvrages aux caractères bien différents et en lui construisant une nouvelle vie» (ibidem, par. 10). De façon nettement plus explicite, Paul Scott affirme que les Mémoires de Choisy «have been taken at face value and wrongly treated as autobiographical» (P. Scott, Autbenticity and Textual Transvestism in the Memoirs of the Abbé de Choisy, "French Studies" 69, 1, 2015, p. 15). Deux des plus importants biographes de l'abbé de Choisy font le choix, quant à eux, de lire les Mémoires comme une confession avant tout autobiographique: D.V.D. Cruysse, L'abbé de Choisy: androgyne et libertin, Paris, Fayard, 1995 et G. Reynes, L'abbé de Choisy, ou L'ingénu libertin, Paris, Presses de la Renaissance, 1983. Si Paul Scott a raison de souligner que tout récit, même s'il se présente comme autobiographique, mêle continuellement le vrai et le fictif, sans doute estil périlleux, en même temps, de faire des Mémoires de Choisy «a literary smokescreen concealing their author's latent same-sex desires» (P. Scott, op. cit., p. 29). Dans le présent article, il sera moins question de savoir si les Mémoires de Choisy sont authentiquement vrais que d'étudier les complexes dispositifs de mise en scène de soi qu'ils déploient. 
sérieux et du rigorisme des onze volumes qu'il consacre à l'histoire ecclésiastique, les Mémoires de Choisy obéissent à un dessein qui confond secret et rumeur, de même qu'ils brouillent la frontière entre le privé et le public. L'abbé de Choisy décrit ouvertement dans ses Mémoires ses aventures travesties, fredaines prétendument entamées dans sa jeunesse $e^{4}$, sous les bons soins de sa mère, femme de cour et précieuse, puis poursuivies jusqu'à sa mort: «ma mère, par une fausse tendresse, m'a élevé comme une demoiselle: le moyen de faire de cela un grand homme! ${ }^{5}$. De façon contradictoire, ces frasques et déguisements androgynes, choquant les mœurs publiques et tout à fait indignes d'un représentant de la foi, sont présentés, à petite distance des grands évènements politiques dont il est témoin, dans de menus détails et sans la moindre atténuation de principe ${ }^{6}$. Ce double projet d'écriture de soi et de l'histoire mobilise et travaille différents régimes de mémoire, autant personnelle que collective. Le présent du récit se heurte au temps de l'histoire, de la même façon que la fiction bouscule le réel, si bien que la représentation du passé coïncide souvent, jusqu'à s'y assimiler, avec la représentation de soi. Chez Choisy, tout fonctionne selon un double paradigme qui oppose, mêle et fédère histoire et fiction. Ces catégories modernes, aux délimitations poreuses et incertaines sous l'Ancien Régime, servent aussi une entreprise plus souterraine, qui refuse cependant de se penser en oppositions binaires et sclérosantes: le travestisme revendiqué de Choisy est partagé, de manière plutôt inattendue, à visage découvert.

L'entrecroisement du féminin et du masculin dans les Mémoires de Choisy impose un dispositif énonciatif hybride, fondé sur le fragment et l'ellipse. La liaison problématique de l'expérience mémorielle et de l'élaboration identitaire se place sous l'égide du travestissement (générique et historique d'abord, identitaire et sexuel ensuite). Le recours à l'écriture personnelle, par l'entremise du genre mémorial, ne témoigne pas tant d'un désir de reproduction mimétique du vécu que celui d'une mise en ordre narrative des évènements passés - comme si l'histoire personnelle s'écrivait selon une mémoire avant tout littéraire. Le récit de soi se compromet ainsi avec le simulacre romanesque. Nous suggérons, dans cet article, que cette instabilité du rapport à soi, corollaire d'une pathologie du rapport à l'autre, et par extension du rapport au temps (personnel et historique), se répercute diversement et obliquement dans le double texte des Mémoires, mais procède d'une même loi: le travestissement.

Les figurations de la subjectivité à l'âge classique, à tout le moins dans le genre des Mémoires, ne sont jamais indépendantes de la configuration et de l'économie collectives où se négocient culturellement les identités, à travers, entre autres instances médiatrices, les destinataires déclarés et les futurs lecteurs. Dans Le Livre avalé, Éric Méchoulan, paraphrasant Roland Barthes, souligne que «les mémoires ne façonnent ou ne témoignent pas pour autant de sujets autonomes, mais d'une "institutionnalisation de la subjectivité"». Dans une société volontiers assujettie au paraître, «ce n'est plus la collectivité qui réfléchit sa valeur dans un être, mais une existence qui publie

(4) Outre les Mémoires, aucun témoignage contemporain ne confirme (ou n'infirme) le penchant que Choisy met en scène. Ce silence démesuré, y compris celui de ses amis les plus proches, est assurément suspect puisqu'une telle pratique n'est pas de celles que l'on met en sourdine.

(5) F.-T. de Choisy, Mémoires de l'abbé de Choisy cit., p. 55.

(6) L'emmêlement du récit de la res publica et de l'histoire personnelle du mémorialiste n'est évidemment pas sans exemple, souvent dans une logique analogue de celle des Vies parallèles de Plutarque, mais la pratique varie beaucoup sous l'Ancien Régime. Ce qui l'est davantage, c'est la projection aussi brusque d'une vie reflétée dans le miroir de l'autre.

(7) É. Méchoulan, Le Livre avalé. De la littérature entre mémoire et culture, Montréal, Presses de l'Université de Montréal, 2004, p. 106. 
les valeurs (ou les non-valeurs) du monde» ${ }^{8}$. Il apparaît alors aventureux de lier son destin personnel, à bien des égards scandaleux et non exemplaire, à celui, grandissime et apparemment intouchable, du roi. Les mémorialistes, en effet, occupent le plus souvent les marges officieuses de l'histoire - la plupart des Mémoires étant écrits dans une position de retrait involontaire suite à une disgrâce. Choisy ajoute à cette situation sociale de périphérie - il se retire en effet au Séminaire des missions étrangères après son retour de Siam -, mais non pas immuable, la marginalité sexuelle. Cette double contrainte qui l'éloigne toujours plus du centre légitimant (c'est-à-dire la cour) s'accompagne d'une mise en scène de soi ambiguë, travestissant les codes de l'écriture de l'histoire et les hiérarchies sexuelles dominantes.

\section{La représentation travestie de l'bistoire. Entre le personnel et le collectif}

Le premier morceau du diptyque bigarré des Mémoires de l'abbé de Choisy s'ouvre à la façon traditionnelle des mémorialistes de l'âge classique et réemploie la plupart des topiques du genre'. L'historien efface sa singularité personnelle et adopte un ton pour le moins désintéressé: «ce n'est point un vain désir de gloire historique qui me met la plume à la main. Je n'attends de mon ouvrage ni honneur ni profit; j'écris pour ma propre satisfaction» ${ }^{10}$. Cette stratégie inaugurale mettant le sujet écrivant à distance de son objet est rapidement confondue, Choisy érigeant la pratique de l'histoire au statut d'œuvre pédagogique et de miroir révélant la vérité:

Je regarde uniquement l'instruction du prochain, et crois que l'histoire est la meilleure et la plus sûre manière d'apprendre aux princes de la terre des vérités quelques fois dures, qu'on n'oserait leur dire autrement ${ }^{11}$. Ils voient, dans ce miroir des choses passées, que la vérité s'y développe tout entière, que les plus puissants rois n'y sont pas épargnés que les moindres de leurs sujets; et que si on y célèbre leurs vertus, leurs vices et même leurs moindres défauts n'y sont pas oubliés ${ }^{12}$.

Se décrétant juge impartial ${ }^{13}$, incorruptible malgré l'économie des dépendances qui régente les échanges mondains, Choisy subvertit la posture déférente du mémorialiste prompt aux louanges serviles, pour lui préférer celle, rigoureusement objective puisque factuelle, de portraitiste courtisan dont la principale ambition est la recherche de la vérité ${ }^{14}$. Aucune vie, aussi illustre soit-elle, n'est exempte d'imper-

(8) Ibidem, p. 90.

(9) Voir E. Lesne, La poétique des Mémoires (1650-1680), Paris, Champion, 1996.

(10) F.-T. de Choisy, Mémoires de l'abbé de Choisy cit., p. 25.

(11) Vialleton remarque que l'ouverture des Mémoires de Louis XIV emprunte beaucoup d'éléments textuels à l'éloge de Bossuet par Choisy, l'une des particularités, entre autres, laissant croire que les Mémoires seraient, peut-être, une entreprise commerciale et diffamatoire (J.-Y. Vialleton, La nouvelle diffamatoire dans la France de l'âge classique cit., par. 11-13). Le phénomène de montage de textes déjà publiés, qui instruit à coup sûr la poétique des Mémoires de Choisy, n'est cependant pas à lui seul suffisant pour lui en révoquer et l'attribution et l'originalité.

(12) F.-T. de Choisy, Mémoires de l'abbé de Choisy cit., pp. 25-26.

(13) Choisy se méfie des liens destructeurs qui lient admiration personnelle et objectivité historique: «Louis lui-même, tout grand qu'il est, ne me tentera pas: quelque faible que j'aie à son égard, la vérité me soutiendra, l'amour du vrai triomphera en moi de tous les autres amours» (ibidem, p. 39).

(14) Plusieurs fois, Choisy revient sur cette impartialité autoproclamée: «Je n'oublierai, s'il m’est possible, aucune de ses vertus; mais aussi je n'oublierai pas ses défauts» (ibidem, p. 27); «Mais je m'arrête tout court, et je trouverai dans la suite de ces Mémoires assez d'occasions de rappeler les dits mémorables de mon héros, que j'estime tel, malgré les fautes qu'il a faites, et qu'il s'est reprochées à lui-même» (ibidem, p. 43). 
fections et d'erreurs. Ainsi Choisy, refusant tout maquillage complaisant du portrait louis-quatorzien, commence l'histoire du roi par le récit contrasté de «deux fautes irréparables $\gg^{15}$ commises durant son règne, l'une personnelle, l'autre militaire; «la première, de n'avoir pas passé le Rhin à la nage après le comte de Guiche» ${ }^{16}$, la deuxième «de n'avoir pas attaqué le prince d'Orange sur la contrescarpe de Valenciennes» ${ }^{17}$. Paradoxalement, la remémoration de la première de ces fautes, somme toute incidente, est aussi l'évènement personnel le plus mémorable et bouleversant de l'existence de l'abbé, c'est-à-dire la mort de M. de Longueville ${ }^{18}$, funeste mais glorieuse: «mais passerais-je si légèrement sur la chose de ma vie qui m'a le plus touché?» ${ }^{19}$. Inoubliable et superlative, cette scène tragique l'est peut-être d'abord parce qu'elle se rapporte à un «ami très particulier» ${ }^{20}$. Ce qui mérite d'être conservé dans la mémoire, plus d'ailleurs que l'évènement lui-même, s'avère les passions que cette mort fameuse a excitées chez l'abbé inexpérimenté:

ce qui est fort singulier, j'étais encore jeune, grand joueur, assez peu attaché à mes devoirs ecclésiastiques (à peine étais-je tonsuré); et cependant j'allai m'enfermer dans une hutte de feuilles [...] et je priai Dieu pour M. de Longueville, à genoux, avec des larmes et une contrition de cœur que je voudrais bien avoir pour mes péchés ${ }^{21}$.

L'extraordinaire de la mort de M. de Longueville est subsumé dans l'extraordinaire de la réaction pieuse de Choisy, mise en scène avec emphase et pathétique. Ainsi l'Histoire au sens fort est rabattue sur l'anecdotique. Le souvenir personnel, doublement mis à distance dans le temps et l'écrit, permet aussi l'élévation soudaine de Choisy, passant de personnage historique secondaire à véritable exemple de religiosité.

Parmi les anathèmes du discours critique sur l'écriture de l'histoire telle que pratiquée au XVII ${ }^{\mathrm{e}}$ siècle émerge une constante: l'exigence de vérité. Pour Choisy, l'accès à la vérité implique d'être immune à toute forme de flagornerie. Il importe aussi, sinon plus, de montrer «[c]es ombres, [c] es taches dans le soleil» ${ }^{22}$, rendant l'ensemble plus cohésif, comme en témoignent les deux micro-récits négatifs placés au seuil du portrait royal. Plus significativement encore, cette vérité supposée de l'histoire surgit dans la contamination constante des mémoires personnelle et collective:

Au reste, j'avertis le lecteur qu'en écrivant la vie du Roi j'écrirai aussi la mienne, à mesure que je me souviendrai de ce qui m'est arrivé. Ce sera un beau contraste, mais cela me réjouira; et je veux bien courre le risque qu'on dise: Il joint à tous propos les louanges d'un fat à celles d'un héros. Ce n'est pas que j'aie envie de me louer; mais, en parlant de soi, on y tombe sans y penser ${ }^{23}$.

Le contraste entre une vie glorieuse et une vie scandaleuse sert, d'une part, le projet pédagogique d'ensemble - Louis XIV sortira grandi de la comparaison -, mais permet, d'autre part, de penser deux régimes à la fois divergents et irréductibles du sou-

(15) Ibidem, p. 44.

(16) Ibidem.

(17) Ibidem, p. 47.

(18) Il s'agit de Charles-Paris d'Orléans, duc de Longueville, mort de façon tragique pendant le passage du Rhin en 1672.

(19) Ibidem, p. 45.

(20) Ibidem.

(21) Ibidem, p. 47.

(22) Ibidem, p. 44.

(23) Ibidem, p. 32. 
venir, l'un collectif, l'autre personnel. Cette mémoire hybride, dont le mode opératoire embrouille représentation du passé et puissance imaginative, a pour principe moteur, nous l'avons dit, le fragment et l'ellipse. Laissant «la grande histoire de son règne» ${ }^{24}$ aux historiographes officiels, plus compétents en la matière, le projet de Choisy, s'il vise en définitive l'intelligence du général, se situe néanmoins au niveau du particulier et du mondain: «je ne m'attache donc qu'aux particularités de la vie du Roi; je tâcherai de le suivre dans ses conseils avec ses ministres, dans ses cabinets avec ses amis» ${ }^{25}$. Le fonctionnement de la mémoire, en ce sens, souscrit tout à fait au «point de vue partiel, personnel et privilégié» ${ }^{26}$ du mémorialiste classique. Autrement dit, l'écriture personnelle de l'histoire est un «discours du singulier» ${ }^{27}$, à la fois dans l'objet de l'énonciation et dans la manière de l'énoncer. En revanche, la vaporisation des souvenirs personnels dans le récit qu'il donne du règne de Louis XIV se remarque de manière parcellaire dans l'œuvre mémoriale, même si l'avertissement inaugural laissait entrapercevoir des liens continus et tranchés entre une vie et l'autre. L'entrelacement du personnel dans le collectif n'obéit pas à des règles poétiques précises ${ }^{28}$, et semble plutôt régi par le désordre et la digression heureuse. Cette mémoire décousue, différée et mobile se double aussi d'une expression du moi mouvante et élastique.

L'une des principales modalités d'inscription de la mémoire personnelle dans l'histoire collective est le ressouvenir, aléatoire mais irrésistible. La stratégie de l'hommage aux grands que l'incipit ménage résiste pourtant assez mal au modus operandi de la mémoire personnelle, sujette à la digression continuelle: «cela me fait souvenir que, pendant que je travaillais à l'Histoire de Charles VI, le duc de Bourgogne, à peine sorti de l'enfance, me dit un jour ces paroles: "Comment vous y prendrez-vous pour dire que ce roi était fou?" $\gg^{29}$. Sa réponse, sèche et sans appel, témoigne de la puissance de vérité de l'écriture: «je dirai qu'il était fou» ${ }^{30}$. Dire l'évènement suffit, en somme, pour l'avérer. Choisy, peu modeste en surdéterminant la place de son œuvre historienne passée, met aussi en abyme un autre type de mémoire: la mémoire livresque. À la fois justification de son mérite littéraire et archive historique, le recours aux livres motive le transit entre le singulier et le général. Le mémorialiste effeuille son récit de références à ses anciens textes, notamment le Journal du voyage de Siam ${ }^{31}$ dont il dit offrir des suppléments inédits au sein des Mémoires de Louis XIV: «je marquerai beaucoup de petites particularités fort ignorées du public; je tâcherai même de ne rien dire de ce qui est dans mon journal» ${ }^{32}$. L'insertion de ce fragment ${ }^{33}$ au contenu

(24) Ibidem, p. 27

(25) Ibidem.

(26) F. Charbonneau, L'écriture de l'bistoire. Saint-Simon et quelques mémorialistes, "Revue d'histoire littéraire de la France" 102, 2, 2002, p. 199.

(27) «Non seulement ils disent le singulier - ce qui ne s'est produit qu'une fois -, mais ils le disent sous un angle singulier, accessible à leurs seuls auteurs» (ibidem).

(28) Les douze livres qui composent les Mémoires pour servir à l'bistoire de Louis XIV, découpe que l'on doit aux éditeurs et non pas à Choisy lui-même, n'accueillent pas systématiquement des digressions personnelles. Les livres I-IV sont inachevés, le mémorialiste s'arrêtant à l'année 1661, et contiennent beaucoup de souvenirs personnels. Le livre $V$ débute à la révocation de l'Édit de Nantes et mène à l'ambassade de Siam (livre XI). Les livres finaux (VII-XII) des Mémoires de Louis XIV, plus touffus historiquement, sont par ailleurs beaucoup plus objectifs dans le ton de l'écriture de l'histoire que l'incipit insolent et hardi, où Choisy souligne pourtant plusieurs fois qu'il y aura bel et bien histoire mêlée.

(29) F.-T. de Choisy, Mémoires de l'abbé de Choisy cit., p. 26.

(30) Ibidem.

(31) F.-T. de Choisy, Journal du voyage de Siam, éd. D. Van der Cruysse, Paris, 1687; Fayard, 1995 [1687].

(32) F.-T. de Choisy, Mémoires de l'abbé de Choisy cit., p. 217.

(33) Placées à mi-chemin du sixième livre, ces notes supplémentaires au Voyage de Siam se poursuivent sur une vingtaine de pages où le primat est accordé à l'autobiographie siamoise. Notons au passage que ce 
moins original que prétendu problématise les rapports entre mémoire personnelle et mémoire collective. Le fragment sur le Siam intervient après un commentaire sur la révocation de l'Édit de Nantes, et rien ne motive, structurellement, l'ajout de cette longue digression personnelle. Cet aparté détonne puisque Choisy se fixe l'objectif, clairement défini mais diversement atteint, de suivre le Roi pas à pas: «je vous promets pourtant bien sérieusement de vous entretenir presque toujours du Roi, ce sera ma base continue» ${ }^{34}$. Choisy indique à plusieurs reprises l'ingrat de la tâche du mémorialiste, à qui l'espace manque pour évoquer toute la richesse de l'histoire d'un si grand personnage. Ici, il se plaint qu'il «ne finirai[t] pas si [il] mettai[t] ici tout ce qui [lui] revient à la mémoire sur un si beau sujet» ${ }^{35}$, là qu'il doit «[s'] arrête[r] tout court» ${ }^{36}$. Il s'accorde néanmoins plus d'une vingtaine de pages pour raconter son voyage asiatique, nombre de loin supérieur à tout autre évènement rapporté par l'abbé: «me voici arrivé à une affaire où l'on me pardonnera bien si je m'étends plus que de coutume: c'est l'affaire de Siam ${ }^{37}$. La prolixité de la mémoire personnelle se confronte à l'insuffisance du discours historique, incapable en définitive d'épuiser l'évènement.

La méthodologie de Choisy en matière d'écriture de l'histoire place le sujet écrivant au cœur de la recherche des sources. En effet, dans le Journal de Siam, que les Mémoires de Louis XIV ${ }^{8}$ prolongent, la dimension journalistique - méthode de l'interrogatoire et croisement des récits de référence suite à l'enquête - du discours historique est déjà soulignée: «j'écrirai tous les soirs ce que j'aurai vu, ce qui s'appelle vu. J'écrirai ce qu'on m'aura dit, et marquerai le nom et les qualités de ceux qui m'auront dit quelque chose, afin que vous ayez plus ou moins d'égard à leur témoignage. Je n'exagérerai point: toujours devant les yeux l'exacte vérité» ${ }^{39}$. La manière pour le moins communautaire dont procède Choisy pour écrire ses Mémoires, c'est-à-dire en agrégeant différentes cellules discursives provenant de sources aussi diverses que lointaines, mime en retour la façon composite qu'a Choisy de se mettre en scène. Le moi représenté, toujours pluriel, se construit semblablement, réunissant sous une identité totalisante et fictionnelle une multitude de récits individuels, disséminés par fragments dans les doubles Mémoires historique et personnel. Cette identité trouble se place également sous le signe de la théâtralité, que l'écriture déguise et révèle à la fois ${ }^{40}$.

souvenir de l'ambassade de Siam est récurrent dans l'œuvre polymorphe de l'abbé de Choisy, puisqu'elle se retrouve aussi, parfois à quelques variantes près, dans le onzième volume de l'Histoire de l'Eglise et dans sa Vie de Salomon.

(34) Ibidem, p. 55.

(35) Ibidem, p. 41.

(36) Ibidem, p. 43.

(37) Ibidem, p. 217

(38) Cette méthode où le ouï-dire constitue l'archive est aussi mentionnée dans les Mémoires de Louis $X I V$ : «Pour moi, voici comme je m’y prends pour écrire mes Mémoires: j'écris d'abord tout ce que je sais par moi-même, et tout ce que ma mère m'a dit; ensuite je fais des questions aux gens par les mains de qui les affaires ont passé, et je les fais sans empressement, avec un air ingénu, de simple curiosité. [...] Je me sers de ce que me dit l'un pour faire parler l'autre; je compare les diverses leçons; et quand plusieurs s'accordent sans s'être concertés, je crois que c'est la vérité» (ibidem, pp. 49-50).

(39) F.-T. de Choisy, Journal du voyage de Siam cit., p. 39.

(40) De manière pour le moins paradoxale et tranchée, Choisy achève la présentation de l'autobiographie siamoise, insérée dans les Mémoires de Louis XIV, par l'aveu suivant: «Or, dans ces Mémoires-ci je ne garderai point de mesures, et dirai tout sans déguisement» (F.-T. de Choisy, Mémoires de l'abbé de Choisy cit., p. 217). Tout dire sans déguisement, puis tout dire sur le déguisement: l'ambiguïté auréole tout à fait le geste de l'écriture, déguisement par excellence. Sur la dimension théâtrale de l'écriture de soi chez Choisy, voir notamment: N. Hammond, All Dressed up... L'abbé de Choisy and the Theatricality of Subversion, "Seventeenth-Century French Studies" 21, 1, 2013, pp. 165-172. 
Si tout, ou presque, sépare les vies parallèles de Louis XIV et de l'abbé de Choisy, une dominante s'observe chez l'un et chez l'autre: la propension aux identités multiples et au déguisement de soi. Choisy, qui avoue d'entrée de jeu «parl[er] de [lui] jusqu'au déboire» ${ }^{41}$, cite une amie qui synthétise la diversité de sa vie tout en la fictionnalisant déjà: «Une dame qui a tout l'esprit du monde a dit que j'avais vécu trois ou quatre vies différentes, homme, femme, toujours dans les extrémités; abîmé ou dans l'étude ou dans les bagatelles; estimable par un courage qui mène au bout du monde, méprisable par une coquetterie de petite fille; et, dans tous ces états différents, toujours gouverné par le plaisir $»^{42}$. Ainsi en va-t-il de même pour le roi: «Il avait naturellement (et il l'a bien mis depuis en pratique) la principale qualité des rois, une profonde dissimulation ${ }^{43}$. Enfin se dessine entre le collectif et le personnel une frontière qui sépare aussi vérité et déguisement.

\section{Dramaturgies de soi. Vérité et déguisement}

Si «les mémoires, plus que tout autre forme d'écrits personnels, se trouvent [...] au cœur de jeux de pouvoir» ${ }^{44}$, il semble que Choisy, a contrario de ses contemporains, n'en fasse pas un usage ou politique ou polémique. Il n'y a chez Choisy ni règlement de comptes ni réécriture de l'histoire dite officielle. Les secrets et particularités que l'on y apprend ne sont pas de l'ordre de l'inimaginable ou de l'extraordinaire, mais se situent au contraire sur le terrain de l'anecdote et du frivole: «Je passe légèrement sur tous les événements publics, on les trouve écrits partout; et je ne veux m'arrêter que sur de certaines choses ignorées du commun des hommes» ${ }^{45}$. Le scandale attendu du travestissement de l'abbé de Choisy est dévoilé à deux reprises dès les premières pages des Mémoires de Louis XIV, et constitue par ailleurs, par projection fictionnelle, l'essentiel de l'autobiographie travestie. Pour distrayante qu'elle soit, cette pratique incongrue d'inversion des genres n'est assurément pas bénigne à l'âge classique. Choisy, homme d'Église tard converti, compromet l'outillage mental hérité de la religion chrétienne ${ }^{46}$, où la différenciation sexuelle triomphe, et brouille de manière radicale les catégories sociales et culturelles conventionnellement admises, puisque «l'interdit religieux de l'inversion des habillements sous-tend et soutient toutes les formes de la division sexuelle qu'elle soit celle des apparences, des rôles, des fonctions ou des fantasmes ${ }^{47}$. La question du travestissement sexuel a beaucoup intéressé la critique, qu'elle soit de l'ordre de la psychanalyse $\mathrm{e}^{48}$ ou issue des gender

(41) F.-T. de Choisy, Mémoires de l'abbé de Choisy cit., p. 55.

(42) Ibidem, p. 33.

(43) Ibidem, p. 79.

(44) E. Méchoulan, Le Livre avalé cit., p. 78.

(45) F.-T. de Choisy, Mémoires de l'abbé de Choisy cit., p. 140.

(46) La référence chrétienne, obligatoire puisque les Mémoires de l'abbé de Choisy habillé en femme sont l'œuvre d'un missionnaire, est souvent fantomatique. Pire, la convocation du religieux sous la plume de Choisy apparaît souvent sacrilège et impie. Ainsi, les curés, moines et autres cardinaux mobilisés dans les Mémoires travestis n'ont pas la vie belle et apparaissent aussi légers que l'abbé. Idem pour les sacrements du mariage, ridiculisés constamment.

(47) N. Pellegrin, Le genre et l'babit. Figures du travestisme féminin sous l'Ancien Régime, article électronique, "Clio. Femmes, genre, histoire" 10, 1999, par. 4, https://journals.openedition.org/clio/252.

(48) Voir par exemple P. Brient, La perversion normale de l'abbé de Choisy, "La clinique lacanienne" 2, 11, 2006, pp. 195-202. Dans un esprit analogue, Mitchell Greenberg soutient que le travestisme de Choisy est une réaction psychique, une résistance à la disciplinarisation des corps promue par l'absolutisme (M. Greenberg, Baroque Bodies: Psychoanalysis and the Culture of French Absolutism, Ithaca et Londres, Cornell University Press, 2001, p. 159). 
studies $^{49}$. Geneviève Reynes évoque par exemple la «bisexualité psychique» ${ }^{50}$ de Choisy pour caractériser l'interchangeabilité des signes genrés dans son œuvre - Choisy étant travesti mais hétérosexuel. Pour Isabelle Billaud, «le masque devient pour l'abbé travesti une identité» ${ }^{51}$. Il faut bien noter en effet la précellence de la métaphore théâtrale ${ }^{52}$ qui accompagne jusqu'au moindre signe la confession de Choisy:

Voilà bien des discours inutiles; vous commandez: j'obéis; mais trouvez bon, Madame, que je ne vous obéisse que par parties; j'écrirai quelque acte de ma comédie, qui n'aura aucune liaison avec le reste; par exemple, il me prend envie de vous conter les grandes et mémorables aventures du faubourg Saint-Marceau ${ }^{53}$.

Le fonctionnement «par parties» de la mémoire personnelle contredit le processus de la mémoire collective, qui procède le plus souvent de manière chronologique et événementielle. Le discours historique, tel que l'ordonnance Choisy dans les Mémoires de Louis XIV, s'entame par les récits croisés de l'enfance du roi et de l'abbé. Dans l'autobiographie travestie, cette prédominance de la chronologie laisse place à l'erratique de la matière romanesque, de la même façon que le mémorialiste passe de portraitiste à dramaturge.

Lorsque Choisy affirme qu'il «laisser[a] tomber de [s]a plume tout ce qui [1]e regardera personnellement $\rangle^{54}$, il convient de nuancer que la vérité personnelle reste souvent cachée sous le masque rassurant de l'habillement en femme (et de sa mise en récit). L'autobiographie travestie, si elle met effectivement à nu une déviance, demeure sur le plan de l'esthétique beaucoup plus que de l'éthique. La réflexion éclair de Choisy sur le travestisme ${ }^{55}$ apparaît même plutôt puérile, axée sur la satisfaction immédiate de plaisirs soi-disant innocents et dénuée de la moindre introspection:

J'ai cherché d'où me vient ce plaisir si bizarre, le voici: le propre de Dieu est d'être aimé, adoré; l'homme, autant que sa faiblesse le permet, ambitionne la même chose; or, comme c'est la beauté qui fait naître l'amour, et qu'elle est ordinairement le partage des femmes, quand il arrive que des hommes ont ou croient avoir quelques traits de beauté qui peuvent les faire aimer ils tâchent de les augmenter par les ajustements des femmes, qui sont fort avantageu ${ }^{56}$.

Le souci de vérité qui règle l'écriture de l'Histoire s'atténue dans l'autobiographie pour donner préséance à l'amour-propre. Ce faisant, l'écriture mémoriale, qui charrie de lourdes exigences internes et des topiques parfois contraignantes,

(49) C'est notamment la position de Nancy Arenberg qui affirme que Choisy «freely satisfies his passion for adorning himself in feminine garb, but he also exploits his gender ambiguity by incorporating illusion into his transvestite performance, thus revealing that the body is a dangerous erotic site» ( $\mathrm{N}$. Arenberg, Mirrors, Cross-dressing and Narcissism in Choisy's "Histoire de Madame la Comtesse des Barres", "Cahiers du dix-septième siècle" 10, 1, 2005, p. 12.

(50) G. Reynes, L'abbé de Choisy, ou L'ingénu libertin cit., p. 187.

(51) I. Billaud, "Une âme de femme dans un corps d'bomme». La représentation du travesti dans les "Mémoires de l'abbé de Choisy", "Lumen" 23, 2004, p. 147.

(52) Frédéric Charbonneau insiste sur la dualité théâtre/sexualité: «le théâtre et le sexe sont pour Choisy dans un rapport circulaire, l'un menant à l'autre en une sorte de vertige» (F. Charbonneau, Sexes hypocrites. Le théâtre des corps chez Jean-Jacques Bouchard et l'abbé de Choisy, "Etudes françaises" 34, 1, 1998, p. 119).

(53) F.-T. de Choisy, Mémoires de l'abbé de Choisy cit., p. 431.

(54) Ibidem, p. 48.

(55) Joseph Harris note d'ailleurs que le raisonnement de Choisy, qui décortique à la va-vite son inclination au travestisme, est une façon d'effacer l'anomalie derrière l'universel: «Yet if Choisy relativizes his own tastes by acknowledging them as effects of his own upbringing, he also attempts to universalize them, suggesting that anyone would be susceptible to such influences» (J. Harris, Hidden Agendas: Cross-Dressing in $17^{\text {th }}$-Century France, Tübingen, Narr, 2005, p. 213).

(56) F.-T. de Choisy, Mémoires de l'abbé de Choisy cit., p. 435. 
semble insuffisante pour accueillir pleinement la mise en spectacle du «moi». Les personnages, presque chosifiés pour accréditer la marginalité de l'abbé, ne sont au final que des faire-valoir, le plus souvent passifs, servant le seul rehaussement de l'orgueil: «je répondais à sa tendresse de toute la mienne; mais quoique je l'aimasse beaucoup, je m'aimais encore davantage, et ne songeais qu'à plaire au genre humain» ${ }^{57}$. S'il est vrai que «nous nous aimons toujours mieux que nous n'aimons les autres $\gg^{58}$, cet amour, dans l'imaginaire dramaturgique de l'autobiographie travestie, transite toujours par la représentation. Pour que le masque corresponde finalement à l'identité, il est nécessaire que la théâtralisation de soi devienne constante. Les Premières intrigues de l'abbé de Choisy sous le nom de madame de Sancy, le premier fragment de l'autobiographie travestie, contiennent en condensé cette surenchère progressive de la représentation. Choisy, résidant alors dans le faubourg Saint-Marceau, tient salon avec ses voisines. D'intrigues en galanteries, Choisy déguisé en madame de Sancy s'entiche de Charlotte, jeune fille qu'il vêtit tout à fait à l'inverse de son sexe: «je fis habiller mademoiselle Charlotte en garçon, je louai un habit complet, fort propre, avec une belle perruque; c'était un fort joli cavalier» ${ }^{59}$. Cette simplification caricaturale du rapport masculin/féminin, où seul l'habit marque la différenciation sexuelle, conforte l'idée selon laquelle le travestissement de Choisy relève surtout de la puérilité et de la coquetterie. Mais le double travestissement, confiné jusqu'alors dans l'espace privé, ne suffit plus: la magnificence de cette personnalité-spectacle a aussi besoin de spectateurs ${ }^{60}$. Suivent les sorties mondaines, l'opéra et le bal masqué. À la représentation théâtrale du travesti s'ajoute aussi sa déclinaison picturale: Choisy fait peindre son portrait en madame de Sancy, celui de Charlotte en M. de Maulny. Ces portraits qui dédoublent l'être ne sont pas d'usage personnel et supposent la médiation de l'autre pour qu'ils s'actualisent pleinement: «quand les deux portraits furent faits, et dans de belles bordures, on les pendit dans mon cabinet l'un auprès de l'autre, et chacun disait: "Voilà un beau couple; il faudrait les marier; ils s'aimeraient bien" $\gg^{61}$. Cette scénographie de soi, fondée sur la surabondance baroque de l'illusion théâtrale, culmine dans la scène du mariage entre travestis et dans la consommation, conservatrice celle-là, de la fausse union: «c'est alors que nous nous abandonnâmes à la joie, sans sortir des bornes de l'hon-

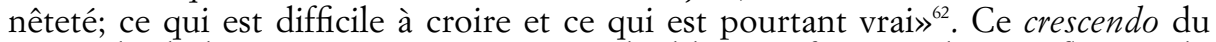
spectacle de l'extériorité travestie ne se double toutefois pas d'une réflexion du même ordre sur l'intériorité:

C'est une étrange chose qu'une habitude d'enfance, il est impossible de s'en défaire: ma mère, presque en naissant, m'a accoutumé aux habillements des femmes; j'ai continué à m'en servir dans ma jeunesse; j'ai joué la comédie cinq mois durant sur le théâtre d'une grande ville, comme une fille; tout le monde y était trompé $e^{63}$.

(57) Ibidem, p. 443.

(58) Ibidem, p. 435.

(59) Ibidem, p. 444.

(60) Dominique Bertrand rapproche le caractère spectaculaire du travesti à celui de la figure royale: «la femme-spectacle que Choisy met en œuvre à travers ses jeux de travesti mime, sur une scène royale secondaire, voire marginale, les mécanismes de la royauté-spectacle de Louis XIV» (D. Bertrand, Fictions et Mémoires de l'androgynie: les écritures croisées de l'abbé de Choisy, in A. Montandon et M. Véga-Ritter (dir.), L'Un(e) miroir de l'autre, Clermont-Ferrand, Université Blaise Pascal, 1998, p. 159).

(61) F.-T. de Choisy, Mémoires de l'abbé de Choisy cit., p. 446.

(62) Ibidem, p. 448.

(63) Ibidem, p. 431. 
Le mémorialiste-comédien se contente au fond du succès durable de son illusion. La variabilité du moi renvoie au singulier de l'Histoire, l'un étant le miroir renversé de l'autre. L'immutabilité du temps historique s'oppose, en quelque sorte, à l'instabilité du temps personnel.

Le travestisme de Choisy, s'il problématise de manière frontale la hiérarchisation des sexes et des pouvoirs sous l'Ancien Régime, intéresse aussi les mécanismes de l'écriture de l'histoire. Dans les Mémoires de Louis XIV, Choisy mentionne que la marche de l'Histoire a pour principal moteur les intrigues féminines, au demeurant minuscules et oiseuses: «les plus grands événements ont presque toujours pour principe des bagatelles, des puérilités, et des tracasseries de femmes ${ }^{64}$. Le rapetissement de l'histoire dans l'infime des tractations mondaines, ou à l'inverse le grandissement du particulier dans l'histoire collective, a néanmoins pour loi commune la présence, autrement spectrale et anecdotique, du féminin. Cette représentation de l'histoire où prime le négligeable se mire également dans l'incipit de l'autobiographie travestie:

Vous m'ordonnez, Madame, d'écrire l'histoire de ma vie; en vérité vous n'y songez pas. Vous n'y verrez assurément ni villes prises ni batailles gagnées; la politique n'y brillera pas plus que la guerre. Bagatelles, petits plaisirs, enfantillages, ne vous attendez pas à autre chose; un naturel assez heureux, des inclinations douces, rien de noir dans l'esprit, joie partout, envie de plaire, passions vives, défauts dans un homme, vertus du beau sexe, vous en serez honteuse en lisant, que serai-je donc en l'écrivant ${ }^{65}$.

Choisy programme aussi la réception de son texte tout en demeurant implicite sur les conditions de sa production. Le passage obligé de la posture révérencieuse du mémorialiste impose dans le même souffle une figure lectoriale honteuse, complice du secret et des péripéties travesties de l'abbé. La responsabilité ainsi déléguée à la marquise de Lambert, destinataire nommée des Mémoires, absout à l'avance l'audace du mémorialiste qui, lui, se soumet docilement à ses obligations. Étant donné la pratique biscornue de l'habillement en femme et des médisances qu'elle susciterait forcément, il aurait été attendu que la démarche soit davantage du côté de l'apologétique que de la dissémination, presque candide et imprudente, de ses propres scandales. Telle est du moins la stratégie employée par les libertins du XVII ${ }^{\mathrm{e}}$ siècle, dont les écrits font un usage massif de l'équivoque et de la dissimulation ${ }^{66}$. Il y a au contraire chez Choisy une évidente urgence à décrire avec faste cette aventure travestie, réifiée selon un canevas narratif à peine altéré de fois en fois - deux récits croisés, mais inversés chronologiquement dans la disposition fictionnelle, construisent l'autobiographie travestie (madame de Sancy et la comtesse des Barres). En outre, la représentation de soi, continuellement saturée de signes, peut exploser à force d'exubérances. «Je me donnais trop en spectacle» ${ }^{67}$, écrit Choisy dans une innocente lucidité. Ce qui fait tenir ensemble les diverses composantes de l'identité de l'abbé, c'est précisément ce principe de la sérialisation du souvenir et de sa mise en récit.

(64) Ibidem, p. 401.

(65) Ibidem, p. 431.

(66) Sur les stratégies d'écriture du «moi» libertin, voir particulièrement L. Tricoche-Rauline, Identité(s) libertine(s). L'écriture personnelle ou la création de soi, Paris, Champion, 2009.

(67) F.-T. de Choisy, Mémoires de l'abbé de Choisy cit., p. 456. 


\section{Sérialité du souvenir et mémoire plagiaire}

Le singulier de l'histoire collective, qu'un récit officiel totalise, trouve parfois un corollaire inversé dans la mémoire personnelle: la sérialisation du souvenir. La représentation de soi, fondement de la pratique travestie, s'appuie surtout sur la réitération, que la vieillesse n'effacera jamais: «Quand je serai bien vieux, je me ferai lire et relire ces Mémoires, et me rajeunirai en quelque sorte en me rappelant ces temps heureux de la jeunesse, où l'on ne songe qu'à se réjouir» ${ }^{68}$. Le dispositif de l'autobiographie travestie est surtout théâtral et romanesque, comme si Choisy, par l'entremise du genre mémorial, réinventait son passé en le structurant comme une série invariablement répétée de tableaux fictionnels. Si la représentation du passé, ainsi que la conceptualise Choisy dans l'incipit des Mémoires de Louis XIV, a une fonction pédagogique, il semble que l'avis concerne plus le roi que l'abbé qui, d'une rouerie à l'autre, ne retire aucun enseignement de ses fautes. Le travestissement de Choisy opère sur le mode de la répétition inlassable. Véritable leitmotiv narratif, la déclinaison du travestissement n'est l'objet ni d'originalité ni de variation: madame de Sancy est aussi et pareillement la comtesse des Barres, seuls de fins détails séparant au fond l'une de l'autre. Le canevas, digne des comédies espagnoles, est rigoureusement le même: l'habillement en femme s'accompagne toujours de la séduction, puis de la débauche d'une enfant-miroir - Charlotte, mademoiselle Dany, les actrices Montfleury et Mondory, mademoiselle de La Grise et Roselie sont au nombre des exploitées tout à fait substituables. Le désir est le même et l'objet, interchangeable. Chaque historiette s'achève mal, forçant l'abbé travesti à l'exil par le voyage. La principale différence entre les deux épisodes majeurs de l'autobiographie travestie (madame de Sancy et la comtesse des Barres) survient dans la mise au jour du travestissement: le premier est graduel tandis que le deuxième est immédiat et total. Comme l'ont suggéré tour à tour Dominique Bertrand, Frédéric Charbonneau et Isabelle Billaud, il y a effectivement progression dans l'accoutumance du travestissement dans l'entourage de l'abbé de Choisy/madame de Sancy: «ainsi, peu à peu, j'accoutumai le monde à me voir ajusté» ${ }^{69}$. Le déguisement s'apprivoise telle une mode nécessitant du temps pour qu'elle brille. C'est effectivement dans la répétition que se fixe durablement l'identité: «j'étais bonne comédienne, c'était mon premier métier» ${ }^{70}$. Dans cette dynamique, la relation avec l'autre n'existe que pour l'exaltation de l'ego. Choisy se dit d'ailleurs «né pour aimer les comédiennes» ${ }^{71}$. Objet privilégié de convoitise, les comédiennes, des professionnelles du masque, sont en effet tout indiquées pour accueillir et réverbérer le reflet démultiplié de l'abbé de Choisy.

La réécriture du souvenir personnel - qui apparaît en calque à la fois dans les Mémoires de Louis XIV et dans l'autobiographie travestie - engendre corrélativement une identité narrative située à la frontière des rapports temps/répétition. Cette identité, que nous pourrions dire irriguée par une esthétique de la parure, Choisy la reçoit déjà façonnée, la subit plus qu'il ne la génère. Il se dédouane d'abord de toute responsabilité: l'odieux du travestissement est l'œuvre de sa mère. Il cherche ensuite caution du côté de prééminentes personnalités: «les femmes aiment qu'on suive leur avis, et madame de La Fayette se crut engagée à faire approuver dans le monde ce 
qu'elle m'avait conseillé» ${ }^{72}$. Il s'émule enfin dans la figure légitimante de Monsieur, également adepte des jeux travestis:

J'allais au Palais-Royal toutes les fois que Monsieur était à Paris; il me faisait mille amitiés parce que nos inclinations étaient pareilles; il eût bien souhaité pouvoir s'habiller en femme, mais il n'osait, à cause de sa dignité (les princes sont emprisonnés dans leur grandeur); il mettait les soirs des cornettes, des pendants d'oreilles et des mouches, et se contemplait dans les miroirs $^{73}$.

Cette triple distanciation de la pratique travestie contraste avec l'hyperbole et la théâtralité qui accompagnent d'ordinaire l'habillement en femme. Significativement, l'identité sérielle de Choisy est dédoublée dans un passage où le spéculaire domine. Au début de l'Histoire de madame de Sancy, une nouvelle inédite est l'objet d'une conversation de salon: «je lui fis lire une petite histoire qui était dans le Mercure galant du dernier mois, où il était parlé d'un homme de qualité qui voulait être femme à cause qu'il était beau» ${ }^{74}$. On ne mentionne à ce moment ni l'auteur ni le titre du texte. Le troisième fragment de l'autobiographie travestie, sans véritable liaison narrative avec le précédent, s'ouvre quant à lui sur le souvenir de l'Histoire de la marquise-marquis de Banneville: «je ne doute point, madame, que l'histoire de la marquise de Banville ne vous ait fait plaisir: j'ai été ravie de me voir en quelque façon autorisée par l'exemple d'une personne si aimable» ${ }^{75}$. En tous les cas, cette intrusion du fictif dans l'autobiographie, déjà massivement soumise au romanesque, complique encore l'élaboration identitaire puisqu'un exemple fictif - la marquise de Banville - autorise Choisy à donner le sien. L'écriture des doubles Mémoires fonctionne ainsi par strates et montages successifs, l'ensemble textuel procédant de l'addition de fragments épars.

Le principe sériel qui décrit le fonctionnement de la mémoire personnelle se répercute aussi à l'échelle de la mémoire historique dans les Mémoires de Louis XIV. La plupart des Mémoires de l'âge classique sont écrits par des témoins oculaires, la valeur testimoniale accréditant l'écriture. S'il fait parfois l'expérience de l'événement dont il rend compte, Choisy doit le plus souvent profiter de la mémoire des autres. Tantôt il s'autoplagie, ou commente l'histoire selon ouï-dire et rumeurs, tantôt il vole carrément le savoir historique d'autrui: «après cet aveu, je ne crois pas être obligé de m'aller déceler présentement à celui que j'ai volé [M. de Dangeau], et que je prétends voler encore: c'est l'homme du monde le plus volable sur ces sortes de matières. Il a été toute sa vie dans le plus fin de la cour: il a tout su, il a tout vu de ses propres yeux ${ }^{76}$. Les Mémoires de Choisy sont donc travestis jusque dans ses sources. Il se dégage de ces vols successifs l'idée selon laquelle l'histoire n'appartient à personne et à tout le monde à la fois, les seuls critères distinctifs demeurant, au fond, l'éloquence du style et le détournement intéressé des événements historiques. Vol de sources, vol d'identités: collégiale ou plagiaire, l'histoire telle que l'actualise Choisy fait un usage à la fois singulier et profondément ambigu de la mémoire, sujette à des pathologies comme la répétition et la réécriture. Le discours historique se construit sur le disparate et le pluriel, de la même façon que l'expression du moi procède du travestissement pour, en définitive, déterminer une identité aux multiples visages.

(74) Ibidem, p. 436.

(75) Ibidem, p. 471.

(76) Ibidem, p. 173 
La mémoire personnelle et la mémoire historique sont, ultimement, toutes deux à la fois façonnées et tributaires de la littérature. Les Mémoires de l'âge classique, rappelons-le, sont avant tout le récit de la particularité aristocratique, souvent conçus dans la polémique pour restituer l'honneur bafoué du mémorialiste déchu. Il y a alors insertion du privé et du personnel dans le public et le collectif: ces deux régimes de la mémoire ne font pas que coexister dans les Mémoires de Choisy, ils s'embrouillent jusqu'à la confusion des termes. Le discours historique et le discours de soi correspondent en fait au même geste: celui de la construction, éminemment narrative, de l'identité.

ALEX BELLEMARE

Université d'Ottawa 